Supporting Information

for

\title{
FIXED-BED ADSORPTION OF CIPROFLOXACIN ONTO BENTONITE CLAY: CHARACTERIZATION, MATHEMATICAL MODELING, AND DFT-BASED CALCULATIONS
}

Raissa Antonellia ${ }^{*}$, Geoffroy Roger Pointer Malpass ${ }^{b}$, Meuris Gurgel Carlos da Silva ${ }^{a}$, and Melissa Gurgel Adeodato Vieira ${ }^{a}$

a School of Chemical Engineering, University of Campinas, Albert Einstein, 500, Campinas, São Paulo, 13083-852, Brazil

${ }^{b}$ Department of Chemical Engineering, Federal University of the Triângulo Mineiro, Randolfo Borges Júnior, 1400, Uberaba, Minas Gerais, 38064-200, Brazil

*corresponding author e-mail address: raissaantonelli@hotmail.com 
Table S1. Comparison of parameters of exhaustion adsorption capacities $\left(q_{e}\right)$, breakthrough adsorption capacities $\left(\mathrm{q}_{\mathrm{b}}\right)$, and adsorption capacities $\left(q_{T H}\right)$ and rate constants $\left(k_{T H}\right)$ of Thomas model reported for the fixed-bed adsorption of CIP onto Verde-lodo clay and different adsorbents (conditions: $D$ is the column diameter, $H$ is the bed height, $Q$ is the flow rate, $C_{0}$ is the inlet concentration).

\begin{tabular}{|c|c|c|c|c|c|c|c|c|c|c|c|}
\hline Adsorbent & $\begin{array}{l}D \\
(\mathrm{~cm})\end{array}$ & $\begin{array}{l}H \\
(\mathrm{~cm})\end{array}$ & $\begin{array}{l}Q \\
(\mathrm{~mL} \mathrm{~min}-1)\end{array}$ & $\begin{array}{l}C_{0} \\
\left(\mathrm{mg} \mathrm{L}^{-1}\right)\end{array}$ & $\begin{array}{l}q_{e} \\
\left(\mathrm{mg} \mathrm{g}^{-1}\right)\end{array}$ & $\begin{array}{l}q_{b} \\
\left(\mathrm{mg} \mathrm{g}^{-1}\right)\end{array}$ & $\begin{array}{l}t_{b} \\
(\min )\end{array}$ & $\begin{array}{l}q_{T H} \\
\left(\mathrm{mg} \mathrm{g}^{-1}\right)\end{array}$ & $\begin{array}{l}k_{T H} 10^{4} \\
\left(\mathrm{~L} \mathrm{mg}^{-1} \mathrm{~min}^{-1}\right)\end{array}$ & Error & Reference \\
\hline \multirow[t]{5}{*}{$\mathrm{CVL}_{\text {clay }}{ }^{a}$} & 0.7 & 7.0 & 1.0 & 331 & 27.8 & 4.0 & 34 & 18.6 & 0.48 & $0.934^{e}$ & \multirow[t]{5}{*}{ This work } \\
\hline & & & 0.7 & 331 & 44.1 & 4.3 & 52 & 35.1 & 0.17 & $0.926^{e}$ & \\
\hline & & & 0.4 & 331 & 50.0 & 8.6 & 167 & 36.8 & 0.08 & $0.861^{e}$ & \\
\hline & & & 0.4 & 199 & 40.4 & 6.0 & 189 & 27.8 & 0.11 & $0.875^{e}$ & \\
\hline & & & 0.4 & 67 & 32.8 & 12.6 & 1149 & 28.2 & 0.16 & $0.935^{e}$ & \\
\hline \multirow[t]{4}{*}{$\mathrm{KAC}^{b}$} & 1.0 & 25 & 1.5 & 225 & - & - & 12 & 1.51 & 3.69 & $0.984^{e}$ & \multirow{4}{*}{$\begin{array}{l}\text { Darweesh } \\
\text { and } \\
\text { Ahmed }^{1}\end{array}$} \\
\hline & & & 1.5 & 75 & - & - & 36 & 0.86 & 8.87 & $0.932^{e}$ & \\
\hline & & & 1.0 & 150 & - & - & 25 & 1.68 & 4.60 & $0.953^{e}$ & \\
\hline & & & 0.5 & 150 & - & - & 37 & 2.09 & 4.58 & $0.941^{e}$ & \\
\hline \multirow[t]{6}{*}{$\mathrm{SGAC}^{c}$} & 1.3 & 4.0 & 1.5 & 50 & 15.8 & 1.58 & 120 & 14.4 & 2.05 & $0.900^{e}$ & \multirow{6}{*}{$\begin{array}{l}\text { Gupta and } \\
\text { Garg }{ }^{2}\end{array}$} \\
\hline & & 6.0 & 1.5 & 50 & 12.5 & 1.4 & 180 & - & - & - & \\
\hline & & 8.0 & 1.5 & 50 & 10.8 & 1.2 & 240 & - & - & - & \\
\hline & & 8.0 & 3.0 & 50 & 15.4 & 1.5 & 120 & - & - & - & \\
\hline & & 8.0 & 1.5 & 100 & 19.7 & 2.2 & 180 & - & - & - & \\
\hline & & 8.0 & 1.5 & 20 & 4.9 & 0.6 & 300 & - & - & - & \\
\hline \multirow[t]{3}{*}{ Chi:nGO ${ }^{d}$} & 1.0 & 1.5 & 4.0 & - & - & - & $<1$ & 91 & 5.1 & $0.077^{f}$ & \multirow{3}{*}{$\begin{array}{l}\text { González } \\
\text { et al. }{ }^{3}\end{array}$} \\
\hline & & & 2.0 & & - & - & $<5$ & 90 & 4.6 & $0.054^{f}$ & \\
\hline & & & 1.0 & & - & - & 30 & 53 & 6.9 & $0.105^{f}$ & \\
\hline
\end{tabular}

$\overline{\mathrm{a}} \mathrm{CVL}=$ calcined Verde-lodo, ${ }^{\mathrm{b}} \mathrm{KAC}=$ granular activated carbon, ${ }^{\mathrm{c}} \mathrm{SGAC}=$ activated sludge derived granular activated carbon, ${ }^{\mathrm{d}} \mathrm{Chi}$ :nGO $=$ chitin matrix with graphene oxide nanosheets, ${ }^{\mathrm{e}}$ Error analysis based on coefficient of determination $\left(R^{2}\right),{ }^{\mathrm{f}}$ Error analysis based on root mean square error (RMSE). 
Table S2. Comparison of parameters of exhaustion adsorption capacities $\left(q_{e}\right)$, breakthrough adsorption capacities $\left(q_{b}\right)$, and adsorption capacities $\left(q_{T H}\right)$ and rate constants $\left(k_{T H}\right)$ of Thomas model reported for the fixed-bed adsorption of ciprofloxacin and ofloxacin onto Verde-lodo clay (conditions: $D$ is the column diameter, $H$ is the bed height, $Q$ is the flow rate, $C_{0}$ is the inlet concentration).

\begin{tabular}{|c|c|c|c|c|c|c|c|c|c|c|}
\hline Adsorbat & $\begin{array}{l}D \\
(\mathrm{~cm})\end{array}$ & $\begin{array}{l}H \\
(\mathrm{~cm})\end{array}$ & $\begin{array}{l}Q \\
\left(\mathrm{mLmin}^{-1}\right)\end{array}$ & $\begin{array}{l}C_{0} \\
(\mathrm{mmol} \mathrm{L}-1)\end{array}$ & $\begin{array}{l}q_{e} \\
\left(\mathrm{mmol} \mathrm{g}^{-1}\right)\end{array}$ & $\begin{array}{l}q_{b} \\
\left(\mathrm{mmol} \mathrm{g}^{-1}\right)\end{array}$ & $\begin{array}{l}q_{T H} \\
\left(\mathrm{mmol} \mathrm{g}^{-1}\right)\end{array}$ & $\begin{array}{l}k_{T H} \\
\left(\mathrm{~L} \mathrm{mmol}^{-1} \mathrm{~h}^{-1}\right)\end{array}$ & $R^{2 c}$ & Reference \\
\hline \multirow[t]{5}{*}{$\operatorname{CIP}^{a}$} & 0.7 & 7.0 & 1.0 & 1.0 & 0.084 & 0.012 & 0.056 & 0.961 & 0.934 & This work \\
\hline & & & 0.7 & 1.0 & 0.133 & 0.013 & 0.106 & 0.346 & 0.926 & \\
\hline & & & 0.4 & 1.0 & 0.151 & 0.026 & 0.111 & 0.165 & 0.861 & \\
\hline & & & 0.4 & 0.6 & 0.122 & 0.018 & 0.084 & 0.214 & 0.875 & \\
\hline & & & 0.4 & 0.2 & 0.099 & 0.038 & 0.085 & 0.312 & 0.935 & \\
\hline \multirow[t]{5}{*}{$\mathrm{OFL}^{b}$} & 0.7 & 7.0 & 1.0 & 1.0 & 0.094 & 0.012 & 0.068 & 0.637 & 0.849 & Antonelli et al. ${ }^{4}$ \\
\hline & & & 0.7 & 1.0 & 0.079 & 0.008 & 0.069 & 0.370 & 0.826 & \\
\hline & & & 0.4 & 1.0 & 0.111 & 0.012 & 0.088 & 0.165 & 0.829 & \\
\hline & & & 0.4 & 0.6 & 0.164 & 0.026 & 0.143 & 0.105 & 0.846 & \\
\hline & & & 0.4 & 0.2 & 0.152 & 0.042 & 0.159 & 0.137 & 0.900 & \\
\hline
\end{tabular}

${ }^{\mathrm{a}} \mathrm{CIP}=$ Ciprofloxacin, ${ }^{\mathrm{b}} \mathrm{OFL}=$ Ofloxacin, ${ }^{\mathrm{c}} R^{2}=$ coefficient of determination. 
Table S3. Bond lengths ( $\AA$ ) calculated with optimized geometries obtained by the B3LYP/6-31G(d) method compared with other DFT methods and experimental data found in the literature for ciprofloxacin (CIP) and ofloxacin (OFL)

\begin{tabular}{|c|c|c|c|c|}
\hline Geometry & $\begin{array}{l}\text { B3LYP/6-31G(d) } \\
\text { (This study) }\end{array}$ & $\begin{array}{c}\text { B3LYP/ } \\
\text { LANL2DZ }^{5}\end{array}$ & $\begin{array}{l}\text { mPW1PW91/ } \\
\text { LANL2DZ }^{5}\end{array}$ & $\operatorname{Exp}^{5}$ \\
\hline \multicolumn{5}{|c|}{ CIP } \\
\hline $\mathrm{R}(\mathrm{C} 16-\mathrm{C} 18)$ & 1.40 & 1.41 & 1.41 & 1.37 \\
\hline $\mathrm{R}(\mathrm{C} 20-\mathrm{C} 21)$ & 1.41 & 1.42 & 1.41 & 1.36 \\
\hline $\mathrm{R}(\mathrm{C} 17-\mathrm{F} 24)$ & 1.36 & 1.41 & 1.40 & 1.33 \\
\hline $\mathrm{R}(\mathrm{C} 25-\mathrm{O} 37)$ & 1.23 & 1.26 & 1.25 & 1.29 \\
\hline $\mathrm{R}(\mathrm{C} 20-\mathrm{N} 28)$ & 1.41 & 1.42 & 1.41 & 1.41 \\
\hline $\mathrm{R}(\mathrm{N} 6-\mathrm{C} 16)$ & 1.40 & 1.40 & 1.39 & 1.49 \\
\hline $\mathrm{R}(\mathrm{C} 38-\mathrm{O} 40)$ & 1.35 & 1.38 & 1.37 & 1.34 \\
\hline $\mathrm{R}(\mathrm{C} 38-\mathrm{O} 42)$ & 1.22 & 1.26 & 1.25 & 1.31 \\
\hline $\mathrm{R}(\mathrm{C} 27-\mathrm{N} 28)$ & 1.36 & 1.37 & 1.36 & 1.41 \\
\hline R(N3-C9) & 1.46 & 1.47 & 1.46 & 1.40 \\
\hline $\mathrm{R}(\mathrm{C} 26-\mathrm{C} 38)$ & 1.48 & 1.48 & 1.47 & 1.47 \\
\hline $\mathrm{R}(\mathrm{C} 30-\mathrm{C} 31)$ & 1.51 & 1.53 & 1.52 & 1.53 \\
\hline \multicolumn{5}{|c|}{ OFL } \\
\hline Geometry & $\begin{array}{c}\text { B3LYP/6-31G(d) } \\
\text { (This study) }\end{array}$ & $\begin{array}{c}\text { B3LYP/ } \\
\text { LANL2DZ }^{6}\end{array}$ & $\begin{array}{c}\text { mPW1PW91/ } \\
\text { LANL2DZ }^{6}\end{array}$ & $\operatorname{Exp}^{6}$ \\
\hline $\mathrm{R}(\mathrm{C} 8-\mathrm{O} 12)$ & 1.23 & 1.25 & 1.26 & 1.22 \\
\hline R(C14-O16) & 1.35 & 1.41 & 1.41 & 1.35 \\
\hline $\mathrm{R}(\mathrm{C} 14-\mathrm{O} 15)$ & 1.22 & 1.23 & 1.24 & 1.22 \\
\hline R(O16-H17) & 0.98 & 0.98 & 0.98 & 0.95 \\
\hline $\mathrm{R}(\mathrm{C} 9-\mathrm{C} 10)$ & 1.37 & 1.38 & 1.38 & 1.48 \\
\hline $\mathrm{R}(\mathrm{C} 8-\mathrm{C} 9)$ & 1.47 & 1.47 & 1.47 & 1.47 \\
\hline R(N11-C20) & 1.48 & 1.49 & 1.49 & 1.49 \\
\hline $\mathrm{R}(\mathrm{C} 20-\mathrm{C} 24)$ & 1.53 & 1.53 & 1.53 & 1.52 \\
\hline R(N29-C30) & 1.46 & 1.47 & 1.48 & 1.48 \\
\hline $\mathrm{R}(\mathrm{C} 1-\mathrm{N} 29)$ & 1.40 & 1.39 & 1.39 & 1.45 \\
\hline
\end{tabular}


Fig. S1. Adjustments of Yoon-Nelson, Yan, Thomas, Clark, Dual site diffusion (DualSD), and Instantaneous local equilibrium (ILE) models for the breakthrough curves obtained at (A) $1.0 \mathrm{~mL} \mathrm{~min}^{-1}$ and $1.0 \mathrm{mmol} \mathrm{L}^{-1}$, (B) $0.7 \mathrm{~mL} \mathrm{~min}^{-1}$ and $1.0 \mathrm{mmol} \mathrm{L}^{-1}$, (C) $0.4 \mathrm{~mL} \mathrm{~min}^{-1}$ and $1.0 \mathrm{mmol} \mathrm{L}^{-1}$, (D) $0.4 \mathrm{~mL} \mathrm{~min}^{-1}$ and $0.6 \mathrm{mmol} \mathrm{L}^{-1}$, (E) $0.4 \mathrm{~mL} \mathrm{~min}^{-1}$ and $0.2 \mathrm{mmol} \mathrm{L}^{-1}$, and (F) $0.4 \mathrm{~mL} \mathrm{~min}^{-1}$ and $0.05 \mathrm{mmol} \mathrm{L}^{-1}$.

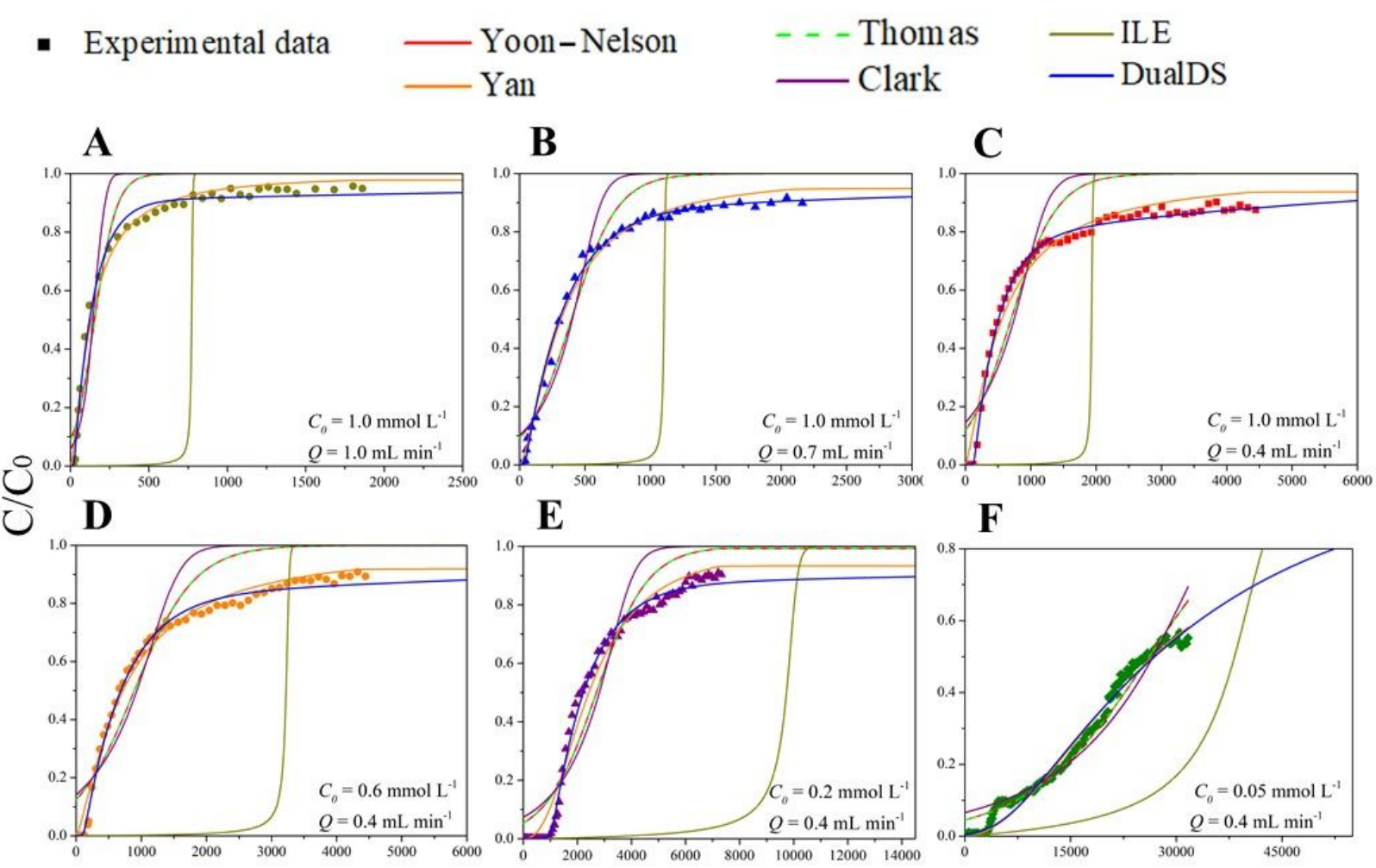

Time (min) 


\section{References}

(1) Darweesh, T. M.; Ahmed, M. J. Adsorption of Ciprofloxacin and Norfloxacin from Aqueous Solution onto Granular Activated Carbon in Fixed Bed Column. Ecotoxicol. Environ. Saf. 2017, 138 (November 2016), 139-145.

https://doi.org/10.1016/j.ecoenv.2016.12.032.

(2) Gupta, A.; Garg, A. Adsorption and Oxidation of Ciprofloxacin in a Fixed Bed Column Using Activated Sludge Derived Activated Carbon. J. Environ. Manage. 2019, 250 (December 2018), 109474. https://doi.org/10.1016/j.jenvman.2019.109474.

(3) González, J. A.; Bafico, J. G.; Villanueva, M. E.; Giorgieri, S. A.; Copello, G. J. Continuous Flow Adsorption of Ciprofloxacin by Using a Nanostructured Chitin/Graphene Oxide Hybrid Material. Carbohydr. Polym. 2018, 188 (November 2017), 213-220. https://doi.org/10.1016/j.carbpol.2018.02.021.

(4) Antonelli, R.; Martins, F. R.; Malpass, G. R. P.; da Silva, M. G. C.; Vieira, M. G. A. Ofloxacin Adsorption by Calcined Verde-Lodo Bentonite Clay: Batch and Fixed Bed System Evaluation. J. Mol. Liq. 2020, 315, 113718.

https://doi.org/10.1016/j.molliq.2020.113718.

(5) Yang, Y.; Gao, H. Theoretical Structure and Vibrational Spectra of Ciprofloxacin: Density Functional Theory Study. Spectrochim. Acta - Part A Mol. Biomol. Spectrosc. 2013, 102, 134-141. https://doi.org/10.1016/j.saa.2012.10.029.

(6) Yang, Y.; Gao, H. Comparison of DFT Methods for Molecular Structure and Vibration Spectra of Ofloxacin Calculations. Spectrochim. Acta - Part A Mol. Biomol. Spectrosc. 2012, 85 (1), 303-309. https://doi.org/10.1016/j.saa.2011.10.019. 